\title{
Effects of mutations in the NMDA receptor GluN1 subunit on binding and dynamics: a computational approach
}

\author{
Zheng Chen ${ }^{1,2}$, W. Bret Church ${ }^{1}$, Karine Bastard ${ }^{1}$, Anthony P. Duff ${ }^{3}$ and Thomas Balle ${ }^{1,2}$ \\ ${ }^{1}$ Sydney Pharmacy School, Faculty of Medicine and Health, The University of Sydney, NSW 2006, Australia \\ ${ }^{2}$ Brain and Mind Centre, The University of Sydney, Camperdown, NSW 2050, Australia \\ ${ }^{3}$ Australian Nuclear Science and Technology Organisation, New Illawarra Road, Lucas Heights NSW 2234, Australia \\ jake.chen@sydney.edu.au
}

N-methyl-D-aspartate receptors (NMDARs) are central to the pathophysiology of neurodegenerative diseases such as schizophrenia [1], however despite significant structural insights of the receptor $[2,3,4,5]$ the importance of mutations in the NMDAR have been poorly described in the literature. Here we present molecular dynamics simulation data combined with modelling and binding free energy calculations to outline the effects of mutations [6] in the GluN1 subunit of the NMDAR on agonist binding affinity and ligandreceptor interactions. Our data demonstrates the changes caused by the positioning of an introduced tyrosine residue at the binding pocket and its associated changes in the conformation upon ligand binding. Furthermore, molecular dynamics simulations demonstrate the changes in ligand environment in the ligand-receptor complex leading to a loss of key interactions and an associated instability of the bound complex. Lastly, binding free energy calculations show that it is no longer energetically favourable for ionic interactions to form and an associated overall increase in Gibbs free energy for ligand binding. These data are important in explaining the changes in behaviour for mutations in the GluN1 ligand binding region and are consistent with previously reported experiments [7]. We are also pursuing experimental approaches to further understand the action of ligand binding.

[1] Coyle JT (2012). NMDA receptor and schizophrenia: a brief history. Schizophr Bull 38: 920-926.

[2] Amin JB, Gochman A, He M, Certain N, \& Wollmuth LP (2021). NMDA Receptors Require Multiple Pre-opening Gating Steps for Efficient Synaptic Activity. Neuron 109: 488-501.e484.

[3] Yu A, \& Lau AY (2018). Glutamate and Glycine Binding to the NMDA Receptor. Structure (London, England : 1993) 26: 1035-1043.e1032.

[4] Tajima N, Karakas E, Grant T, Simorowski N, Diaz-Avalos R, Grigorieff N, et al. (2016). Activation of NMDA receptors and the mechanism of inhibition by ifenprodil. Nature 534: 63-68.

[5] Furukawa H, \& Gouaux E (2003). Mechanisms of activation, inhibition and specificity: crystal structures of the NMDA receptor NR1 ligandbinding core. The EMBO journal 22: 2873-2885.

[6] Zehavi Y, Mandel H, Zehavi A, Rashid MA, Straussberg R, Jabur B, et al. (2017). De novo GRIN1 mutations: An emerging cause of severe early infantile encephalopathy. Eur J Med Genet 60: 317-320.

[7] Skrenkova K, Song J-m, Kortus S, Kolcheva M, Netolicky J, Hemelikova K, et al. (2020). The pathogenic S688Y mutation in the ligand-binding domain of the GluN1 subunit regulates the properties of NMDA receptors. Sci Rep 10: 18576.

Keywords: protein-ligand complexes; receptors; molecular dynamics; ligand binding; mutant receptors 